Marquette University

e-Publications@Marquette

$1-1-2009$

\title{
Phänomenologische und Mundane Reduktion
}

Sebastian Luft

Marquette University, sebastian.luft@marquette.edu

Published version. "Phänomenologische und Mundane Reduktion," in Die Sichtbarkeit des

Unsichtbaren. Eds. Rudolf Bernet and Antje Kapust. Paderborn, DE: Wilhelm Fink, 2009: 57-72.

Publisher Link. (C) 2009 Wilhelm Fink. Used with permission. 


\section{PHÄNOMENOLOGISCHE UND MUNDANE REDUKTION}

Der Kerngedanke der Husserlschen Philosophie, die phänomenologische Reduktion, hat in seiner nahezu 60jährigen Rezeption zahlreiche Deutungen erfahren.' Hierzu haben sicherlich auch Husserls eigene, im Laufe seines Lebens variierenden, teilweise Missverständnis erweckenden Darstellungen beigetragen. Ob nun ,die Reduktion“, allgemein verstanden als grundsätzlicher Titel für die Husserlsche Eingangspforte in und Weg zu letztausweisender wissenschaftlicher Philosophie, von ihren Interpreten gedeutet wurde (um vielleicht die Extreme zu nennen) als ein blinder Voluntarismus ${ }^{2}$, oder als eine aller Verantwortlichkeit sozialen Handelns entbehrender Passivität oder Weltflucht ${ }^{3}$, ja gar als eine Art „Elitarismus“, der ,ausgesprochen aristokratische

1 Die meines Wissens ersten Darstellungen von Husserls Philosophie, in denen die Reduktion als ein zentrales Bestandstück derselben behandelt wird, reichen zurück bis in die späten 20er Jahre; s. etwa von Th. Celms aus dem Jahre 1928 (Celms, Theodor, Der phänomenologische Idealismus Husserls und andere Schriften 1928 - 1943, hg. von Juris Rozenvalds. Frankfurt am Main/ Berlin/ New York/ Paris/ Wien 1993), sowie Mischs Abhandlung über Husserl und Heidegger von 1929/30 (Misch, Georg, Lebensphilosophie und Phänomenologie, Separatdruck aus dem ,.Philosophischen Anzeiger“, Heft 3/4, 1929/30; Photomechanischer Nachdruck Darmstadt 3. Aufl. 1967). V.a. Mischs Abhandlung, die Husserl aufmerksam las und annotierte, war für Husserls Selbstverständnis und sein Bild in der philosophischen Öffentlichkeit sehr wichtig, und sie war es, die ihn ab dem Frühjahr 1930 erneut dazu veranlasste. eine neue Einführungsschrift zu seinem philosophischen System (eine neue Darstellung der Reduktion also) zu verfassen. Vgl. hierzu die Darstellung in der Einleitung des Herausgebers Iso Kern, (in: Husserl, Edmund, Zur Phänomenologie der Intersubjektivität III, (Hua XV), hg. ion Iso Kern, Den Haag 1973, S. XLII - XLVIII, insbes. S. XLVII f.). [Der ursprüngliche und vollständige Titel dieses Beitrages lautete: „Phänomenologische und mundane Reduktion: Die Reduktion als Enthüllung des „Absoluten“ und die Frage nach dem philosophierenden Subjekt", er musste aufgrund der Kopfzeilen-Passung gekürzt werden, Antje Kapust].

Vgl. hierzu von Philip R. Buckley Husserl, Heidegger and the Crisis of Philosophical Responsibility, (Dordrecht/ Boston/ Lancaster 1992, S. 131 - 143, insbes. S. 136). Buckley interpretiert Husserls Philosophie in seiner späten Phase im Ganzen als einen ,.universalen Voluntarismus“, der als einzige Lösungsstrategie aus der Krisis der Neuzeit eine sich selbst absolut verantwortende Aktivität propagiert, hierbei aber gerade der Crux der Neuzeit (einem ins Hektische gesteigerten ,betriebsamem“ Aktivismus) aufs neue verfallend. So unterstellt Buckley Husserls Philosophie auch eine „totalizing tendency“ (S. 138).

Zu dieser meines Erachtens schiefen Interpretation sind insbesondere die Kritiker gekommen. die zu stark an das skeptische Verständnis von epoché angeknüpft haben, indem sie das aus der antiken Skepsis stammende Ideal der epoché in erster Linie mit der ataraxia. der ..Seelenruhe", in Verbindung brachten. Vgl. dagegen aber, ein umfassenderes Verständnis der skeptischen epoché restituierend von Klaus Held „Husserls Rückgang auf das phainómenon und die geschichtliche Stellung der Phänomenologie“ (in: Phänomenologische Forschung 10 (1980), S. 89 - 145). Hier zeigt Held, dass die ataraxía, die in der Tat für die Skeptiker einen Rückzug aus der sozialen und politischen Welt bedeutete, nur eine Ausprägung der epoché ist, und dass man sie mit anderer Akzentsetzung durchaus mit der Fordenung nach politischem Engagement verbinden kann und sogar muss. 
Züge trägt, weil [er] nur Auserwählten zugänglich ist" ${ }^{\star 4}$, so wurde jeweils ein Aspekt herausgegriffen und zu Ungunsten anderer überbewertet. Schließlich hat es nicht an Versuchen gefehlt (auch hierin Husserlsche Motive aufnehmend), die Reduktion mit anderen Denkfiguren der Philosophiegeschichte in Beziehung zu setzen, sowie sie in anderen Ansätzen der phänomenologischen Bewegung, die sich nicht explizit darauf beriefen oder dieses ,transzendentalidealistische" Element ab den Ideen sogar ausdrücklich ablehnten, dingfest $\mathrm{zu}$ machen. ${ }^{5}$

Dabei war es gerade Husserls Überzeugung, dass die Reduktion nicht nur der wichtigste, sondern insbesondere der eigenständigste Bestandteil seiner Philosophie sei, der eine derartige Radikalität bedeutete, dass keine philosophische Denkfigur der Vergangenheit auch nur Vergleichbares unternommen hätte (wenn es auch gewisse Vorstufen dazu gab). Ja, zum Ende seines philosophischen Weges scheint sich für Husserl alles darauf zu konzentrieren, den Sinn der phänomenologischen Reduktion recht zu verstehen; sei es, dass Husserl selbst in immer neuen Anläufen eine restlos überzeugende Einleitung in die Phänomenologie zu verfassen sich vornahm; sei es als Spitze gegenüber seinen Kritikern, die sein ganzes philosophisches Unternehmen in erster Linie deshalb nicht verstanden, weil sie kein richtiges Verständnis der Reduktion hatten. Kein Wunder also, dass die Nachwelt gerade in bezug auf die Deutung dieser ominösen Methode die unterschiedlichsten Interpretationsvorschläge vorgelegt hat.

All das hat dazu beigetragen, den komplexen Grundgedanken der Reduktion zu verwässern, manchmal in einer Weise, dass es dem Begriff selbst nicht anders erging als einem ähnlichen Grundterminus der Husserlschen Phänomenologie, der heute allenthalben auftaucht: dem der Lebenswelt. ${ }^{6}$ Dem aber widerspricht eine rigidere Auslegung, die auf den spezifisch transzendentalphänomenologischen Sinn der Reduktion, der sich aus konkreten erkenntnistheoretischen Problemen in Husserls Frühwerk herleitet, verweist.' So könnte man

4 So Kosing im Lemma „Phänomenologie“, in: Wörterbuch der [marxistisch-leninistischen] Philosophie, S. 401.

5 So hat es etwa Bemühungen gegeben, die Stimmung der Angst in Heideggers Daseinsanalyse als eine (Art von) Reduktion zu bezeichnen; vgl. hierzu von Rudolf Bernet ,Phenomenological Reduction and the Double Life of the Subject“, (in: Reading Heidegger from the Start. Essays in His Earliest Thought, hg. von Theodore Kisiel und John van Buren, New York 1994, S. 245 - 267) und vor ihm von Jean-Luc Marion, Jean-Luc, Étant donné. Essai d'une phénoménologie de la donation (Paris 2. Auf1. 1998, S. 71 - 76). Diese Interpretationen gehen allerdings davon aus, dass man den methodischen Schritt der Reduktion vom Vollzug eines Subjekts isolieren kann, was aus der Sicht des „Subjektivisten“ Husserl unplausibel wäre, insofern die Reduktion ein spezifischer methodischer Schritt innerhalb eines wissenschaftichen Unternehmens ist, das ein sich selbst verantwortendes und aktiv tätiges Subjekt voraussetzt.

6 Cum grano salis kann daher für den Reduktionsbegriff gelten, was Held einmal in bezug auf den der Lebenswelt kritisch feststellt, dass er „,beinahe alle Konturen verloren“ habe.

" Vgl. hierzu die fünf Vorlesungen über "Die Idee der Phänomenologie“ (Husserl, Edmund, Die Idee der Phänomenologie, (Hua II), hg. von Walter Biemel, Den Haag 2. Aufl. 1973). sowie die wichtige Vorlesung von 1906/07 (Husserl, Edmund, Einleitung in die Logik und 
im Überblick über die verschiedenen Interpretationsversuche einerseits den Eindruck gewinnen, dass, wer allzu sehr im Rahmen der Husserlschen Phänomenologie verbleibt, einer, letztlich am ohnehin ,,veralteten“ Subjektivitätsparadigma verhafteten, obsoleten Denkfigur nachhänge, und dass die Phänomenologie ganz gut ohne eine Reduktion auskommen könne. Wer ein solches philosophisches Projekt noch durch eigenes Weiterdenken unterstütze, habe von der modernen Subjektivitäts- und Rationalitätskritik im Wesentlichen nichts verstanden, oder schlimmer, sie ignoriert.

Die andere, ,freiere“ Behandlungsart sieht sich demgegenüber darin berechtigt (übrigens nicht zu Unrecht unter Berufung auf Husserl), den Grundgedanken der Reduktion in einem Maße zu verallgemeinern, dass man von einer ,Reduktion“ überall da sprechen kann, wo es um die Freilegung noch verborgener Schichten im Gefüge der Welt-,,Konstitution“" geht. „,Reduktion“ ereignete sich überall dort, wo etwas noch Radikaleres, sich dem normalen Blick ,zunächst und zumeist“ Entziehendes entdeckt wird. Kann ersteres Vorgehen ein „Reduktionismus der Reduktion“ genannt werden, ist bei letzterem das Gegenteil der Fall. ${ }^{8}$ Die Reduktion wird in einer Allgemeinheit verstanden, die sich verdächtig an Beliebigkeit annähert. Gleichgültig, wie man nun die Reduktion deuten mag, so kann man doch grundsätzlich feststellen, dass Husserl mit diesem Verfahren, mit dem er auf plausible und gar zwingende Weise in die Philosophie einführen wollte, auf nichts weniger hinauswollte, als das sichtbar zu machen, was sich dem normalen Blick entzieht; denn zu Anfang ist „die Phänomenologie" nicht vorgegeben, wäre sie es, bräuchte man kein kompliziertes Verfahren, in sie einzuführen. So kann man zu Recht sagen, dass die Reduktion Husserls Beitrag dazu ist, das primär Unsichtbare sichtbar zu machen, oder um es in seiner Terminologie zu sagen, die natürliche Einstellung über das ihr eigene, jedoch verborgene transzendentale Leben aufzuklären.

Erkenntnislehre, (Hua XXIV), hg. von Ulrich Melle, Dordrecht 1984). Bezeichnenderweise versucht Husserl in seiner späteren Selbstinterpretation die Entdeckung der Reduktion zunehmend früher anzusetzen; schließlich sollen sogar die Logischen Untersuchungen mit einer Reduktion und einem impliziten Wissen um das Korrelationsapriori operiert haben. freilich ohne dass es dem Autor zu diesem Zeitpunkt klar gewesen wäre.

Als Vertreter der ersteren Lesart sind v.a. die Vertreter der Münchner Phänomenologie zu nennen, die sich geradezu dadurch auszeichnen, dass sie die Reduktion und damit die transzendentale Wende Husserls tout court ablehnten. Diese Position wurde im Folgenden z.B. von Philosophen wie Schütz und Patočka übernommen, die Versuche unternahmen, eine Ontologie der Lebenswelt als positive Wissenschaft durchzuführen, also das, worauf Husserl gerade durch die Reduktion geführt wurde, ohne selbige zunächst zu vollziehen. Als aktuelles Beispiel für den zweiten Typ sehe ich Jean-Luc Marion (Étant donné. Essai d'une phénoménologie de la donation, a.a.O.), der ..die Reduktion“ in Husserl. Heidegger und seinem eigenen Denken darstellt, dabei aber (soweit ich sehe) nicht eigens die Frage thematisiert. was Reduktion bedeuten kann, um es überhaupt zu ermöglichen. auch bei Heidegger (und Marion selbst) von Reduktion zu sprechen. So geht es bei ihm, so scheint es mir. auch gar nicht um eine Reduktion selbst, sondern darum, worauf sie reduziert: bei Husserl auf die transzendentale Subjektivität, bei Heidegger auf das Sein und bei Marion schließlich auf das, was er ..reine Gegebenheit“" (donation, givenness) nennt. 
Und doch geht es ihm hierbei um mehr; denn es wird nicht nur die eigentliche Sichtbarmachung des zunächst Unsichtbaren zum Problem; sondern auch das Paradigma der phänomenologischen Beschreibung selbst, das zunächst von einer rigiden Trennung von Sicht- und Unsichtbarem ausging, wird bedeutenden Modifikationen unterworfen, die eminente Konsequenzen für sein philosophisches Projekt haben.

Im Folgenden kann weder beansprucht werden, ein vollständiges Bild der Reduktion zu entwerfen, noch soll es darum gehen, sie angesichts ihrer unbestrittenen Schwierigkeiten auf Messers: Schneide zu verteidigen. Vielmehr wird der Versuch unternommen, zu einem Verständnis der im weiteren Sinne „metaphysischen“ Dimension derselben beizutragen, wenn es denn stimmt, dass die Reduktion, einem Wort Landgrebes zufolge, „Husserls Zugangsweise zur Metaphysik" (freilich eines Husserl zufolge neuen Sinnes) ist". Es geht darum zu explizieren, was mit der Reduktion für Husserl letztlich auf dem Spiel steht. Es verdient der Erwähnung, dass man sich seit einiger Zeit durch neue Veröffentlichungen von Texten aus Husserls Spätwerk, insbesondere der VI. Cartesianischen Meditation und Husserls Anmerkungen dazu ${ }^{10}$ und nicht zuletzt durch den umfangreichen Briefwechsel, einen besseren und umfassenderen Eindruck seiner abschließenden Gedanken machen kann. In diesen späten Reflexionen beginnen sich die Konturen eines phänomenologischen Systems abzuzeichnen, und für Husserl stand aüßer Frage, dass dieser antizipierte Abschluss nicht ohne eine radikalisierte:Darstellung der Reduktion möglich sei. Diese Radikalisierung geht jedoch mit einer (Neu-)Beurteilung oder Neubewertung derselben hinsichtlich des mit ihr verfolgten Ziels bzw. ihrer Absicht einher. Letztere können unter dem zentralen Titel der „Verweltlichung“ zusammengefasst werden. ${ }^{11}$

9 Vgl. von Ludwig Landgrebe, Der Weg der Phänomenologie. Das Problem einer ursprünglichen Erfahrung, (Gütersloh 1978, S. 82 ff.). Ähnliches hat Van Breda bereits im Jahr 1959 ausgedrückt, wenn er die These vertritt, „que, pour Husserl, la problématique de la réduction. dans son sens le plus profond, n'est autre chose que sa découverte de la dimension métaphysique“ (Van Breda, Hermann Leo, „La réduction phénoménologique“, in: Husserl, hg. von M.- A. Bera, Paris 1959, S. 307 - 18, hier S. 308).

10 Ich lese die VI. Cartesianische Meditation als einen genuinen Text Finks, der hauptsächlich eine andere Linie verfolgt als Husserls Denken. Sie soll daher im .Vorstehenden nicht eigens herangezogen werden. Allerdings wird hier und da auf die in den beiden Bänden veröffentlichten Zitate Husserls zurückgegriffen, sofern sich Husserl durch Finks Ausführungen herausgefordert sah, seine Position zu erneut zu bestimmen (siehe Fink, Eugen, VI. Cartesianische Meditation. Teil I. Die Idee einer transzendentalen Methodenlehre, hg. von Hans Ebeling, Jann Holl und Guy van Kerckhoven (Hua Dokumente Bd. II/1), Dordrecht/ Boston/ Lancaster 1988).

1 Vgl. hierzu auch van Kerckhoven 1998, der dieses Thema jedoch unter besonderer Berücksichtigung der Finkschen Überlegungen in seiner VI. Cartesianischen Meditation behandelt (Van Kerckhoven, Guy, Mondazzione e Individuazione. La posta in gioco nella Sesta Meditazione cartesiana di Husserl e Fink, Genua 1998). Van Kerckhovens Abhandlung stellt die erste ausführliche Monographie zum Komplex „VI. CM“ dar. * 
Kann man die „mittlere“ Phase der Husserlschen Bemühungen um die Gestaltung der Reduktion als eine neue Darstellung des Wegs in die Phänomenologie bezeichnen (wobei hier gerade die Pluralisierung in Wege den entscheidenden neuen Schritt bezeichnet ${ }^{12}$ ), so ist die späte Phase vornehmlich bestimmt durch die Versuche der (historischen wie systematischen) Selbstcharakterisierung der Phänomenologie ${ }^{13}$ und damit des ganzen Projekts Phänomenologie als eines solchen. Bedingung hierfür ist, dass ihm die Reduktion selbst sowie ihre Durchführung und ihr Ziel nicht mehr eigentlich fraglich scheinen. Daher wird für Husserl immer stärker die Frage virulent, was eigentlich geschieht, wenn sich ein Individuum in radikaler Vereinzelung zum philosophischen bzw. phänomenologischen Denken erhebt. Die umfassende Frage, die sich für Husserl jede Philosophie, die mit einem Anspruch wie der seinen auftritt, stellen muss (und nur eine solche ist für ihn überhaupt Philosophie zu nennen), ist die nach dem „Zwecksinn“ dieses ganzen Unternehmens, und das ist letztlich, wenn man den ,ganz großen“ Blick wagt, für ihn nichts anderes als des Projekts „Wissenschaft“" in der Geschichte Europas. ${ }^{14}$ Hierzu muss sich die Phänomenologie als universale Kritik der Vernunft wiederum einer Selbstkritik unterziehen. Dies sind hinreichend bekannte Sachverhalte, die Husserl unter den Titel „Phänomenologie der phänomenologischen Reduktion“ oder „Phänomenologie der Phänomenologie“ behandelt. ${ }^{15}$ Einer weit verbreiteten Forschungsauffassung zufolge hat Husserl diese Kritik nie durchgeführt $^{16}$; stimmt dies in bezug auf seine veröffentlichten Schriften, so ermögli-

12 Vgl. hierzu insbesondere Husserl, Edmund, Erste Philosophie (1923/24). Erster Teil: Kritische Ideengeschichte, (Hua VII), hg. von Rudolf Boehm, Den Haag 1956; sowie Husserl, Edmund, Erste Philosophie (1923/24). Zweiter Teil: Theorie der phänomenologischen Redutition, (Hua VIII), hg. von Rudolf Boehm, Den Haag 1959. Vgl. auch die Vorlesung von 1922/23 „Einleitung in die Philosophie“ (hauptsächlich im Konvolut F I 29), die, als Vorläufer von „Erste Philosophie“, untrennbar diesem Projekt zugehört. Diese Vorlesung ist zur Veröffentlichung im Rahmen der Husserliana vorgesehen.

13 Dies ist die Betitelung der Ms.-Signaturgruppe B IV, aus der die wichtigsten diesbezüglichen Texte veröffentlicht sind in den Bänden der VI. Cartesianischen Meditation (Hua Dok. IV/1 und 2, a.a.O.).

14 Diese Themen werden von Husserl bereits in den sog. Kaizo-Artikeln von $1922-24$ (Husserl. Edmund, Aufsätze und Vorträge (1922 - 1937), Beilage X, (Hua XXVII), hg. von Th. Nenon und H. R. Sepp, Dordrecht/Boston/London 1989, S. 3 - 124) berührt, und die späteren Überlegungen müssen als Fortsetzung jener allgemeineren (und für ein weniger fachphilosophisches Publikum verfassten), auf das Wesen der Kultur abzielenden Reflexionen gesehen werden. Auch die Reduktion ist für Husserl letztlich eine ethische - .,erkenntnisethische“ - Frage.

Zur Wendung „Phänomenologie der phänomenologischen Reduktion“ vgl. Erste Philosophie (Hua VIII, a.a.O., 3. Abschnitt, S. 82 - 131). Zur Wendung „Phänomenologie der Phänomenologie" vgl. die VI. CM (a.a.O., S. 9). Letztere Formulienung taucht auch in Husserls Nachlass bisweilen auf, vgl. z.B. B II $6 / 2$ a.

16 Dieser Auffassung ist etwa Landgrebe, und seine Deutung wurde zumeist fraglos übernommen. Eine originelle Deutung vertritt Zahavi, wenn er behauptet, dass diese Selbstkritik durch Husserls Überdenken der Intersubjektivitätsproblematik im Wesentlichen obsolet gemacht wurde (vgl. Zahavi, Dan, Husserl und die transzendentale Intersubjektivität. Eine Antwort auf die sprachpragmatische Kritik, Dordrecht/ Boston/London 1996, S. 99). Diese Interpretation unterschlägt m.E. jedoch die Rolle, die für Husserl das phänomenologisierende Subjekt spielt. 
chen es doch seine nachgelassenen Manuskripte v.a. aus den 30er Jahren, eine solche Selbstkritik zu rekonstruieren. Diese Selbstkritik betrifft nun nicht allein eine Kritik der phänomenologischen Erfahrung, sondern geht auf das Unternehmen „Phänomenologie“"im Ganzen.

Es geht im Rahmen der Selbstkritik der phänomenologischen Erkenntnis um die Frage nach dem philosophierenden Subjekt, welches sich selbst durch Reduktion aus dem Zustand der natürlichen Einstellung erhebt und sich selbst als den (bzw. in sich selbst den) ,unbeteiligten Zuschauer“ hervorbringt. Auch wenn wohl kaum ein Philosoph so viel Bemühungen um diesen ominösen (und gleichzeitig heftig kritisierten ${ }^{17}$ ) Zuschauer aufgewendet hat, so ist doch das Motiv der Unbeteiligtheit der theoría gegenüber der prâxis uralt, und Husserl selbst greift mitunter auf traditionelle Denkfiguren zur Charakterisierung dieses Verhältnisses zurück (z.B. Platon, Augustinus). ${ }^{18}$ Es wird nicht überraschen, wenn sich in der Husserlschen Behandlung dieses Sachverhalts - in der Beziehung zwischen ",natülicher“ und (mit Held zu reden) „,doxakritischer" Einstellung ${ }^{19}$ - ähnliche oder doch analoge Probleme ergeben werden, und sich Husserl mit nolens volens traditionellen Lösungsvorschlägen konfrontiert sieht.

Weil die durch Reduktion erlangte phänomenologische Einstellung, die erst das philosophierende Subjekt vollends als ,unbeteiligten“ Zuschauer etabliert, nicht nur der ursprünglichen natürlichen Einstellung gegenüber radikal anders, sondern auch der objektivistisch-kritischen der Wissenschaft gegenüber höherstufig ist, sieht sich gerade der transzendentale Zuschauer wieder in der Lage, seine eigentümliche Position bestimmen zu müssen, und das ist nur in

das seine „Undeklinierbarkeit“ nicht aufgeben kann (auch wenn es Teil einer Forschungsgemeinschaft ist).

17 So bezeichnet Landgrebe gar einmal diesen „unbeteiligten Zuschauer“ als den wichtigsten Stein des Anstoßes für die Kritik an Husserls transzendentalphilosophischem Projekt, sofern sich hieran der Charakter von Husserls Phänomenologie als Reflexionsphilosophie am deutlichsten ablesen lasse; vgl. von Ludwig Landgrebe, Der Weg der Phänomenologie. Das Problem einer ursprïnglichen Erfahrung, (a.a.O., S. 32).

18

Fink hat in seinem Thatwelt-Aufsatz „Was will die Phänomenologie Edmund Husserls?“ von 1934 etwa die Beziehung zum platonischen Höhlengleichnis und zum thaumázein als Beginn der Philosophie hergestellt (vgl. Fink, Eugen, Studien zur Phänomenologie 1930 - 1939, Den Haag 1966, S. 158 - 164). Wie aus Husserls Einleitungsentwurf dieses Aufsatzes, der von Fink später breiter ausgeführt wurde, hervorgeht, stammt die Anknüpfung an das Höhlengleichnis von Husserl selbst, vgl. K II 2/13 - 15. Auch die thaumázein-Metapher wird von Husserl wieder aufgegriffen im Wiener Vortrag von 1935 (Husserl, Edmund, Die Krisis der Europäischen Wissenschaften und die transzendentale Phänomenologie, (Hua VI), hg. von Walter Biemel, Den Haag 2. Auf. 1962, S. 331). Zu Augustinus vergleiche die Ausführungen von Husserl, in denen er das berühmte Augustinische ,si nemo me quaerat..." als eine frühe Formulierung von vorphilosophischer und philosophischer (reflexiver) Einstellung verwendet (Husserl, Edmund, Zur Phänomenologie des inneren Zeitbewußtseins (1893 - 1917), (Hua X), hg. von Rudolf Boehm, Den Haag 1966, S. 3).

19 Vgl. von Klaus Held, „Husserls These von der Europäisierung der Menschheit“" (in: Jamme, Christoph/ Pöggeler, Otto (Hg.), Phänomenologie im Widerstreit. Zum 50. Todestag Edmund Husserls, Frankfurt am Main 1989, S. 13 - 39, S. 14 ff.). 
Abgrenzung gegen das möglich, was sie wesenhaft nicht ist. Das ist aber gleichbedeutend damit, dass der Zuschauer sich nach seinem „désengagement" von seiner Befangenheit in der natürlichen Einstellung wiederum selbst thematisieren muss, und d.h. sich gegenüber dem ihm Anderen rechtfertigen muss. Das wiederum hat zur Folge, dass er sich „re-engagieren“ muss in die Lebensweise, die er durch die Reduktion gerade ,überwunden“ hat. Gleichzeitig mit diesem Re-engagement muss er also seine Position gegenüber seinem Ausgangspunkt bestimmen. Die Anspielung an das Platonische Höhlengleichnis ist hierbei nicht zufällig. Diese der (mit Hart zu reden ${ }^{20}$ ) „:entripetalen“" Bewegung der Reduktion entgegengesetzte, ,zentrifugale“ Tendenz fungiert bei Husserl unter dem genannten Begriff der Verwieltlichung. Es wird sich zeigen, dass in Wahrheit keine der so nahe liegenden Deutungen der Reduktion dem eigentlichen Verständnis derselben so sehr schaden kann wie die scheinbar so evidente Anknüpfung an diese Platonische Allegorie, der sich unglücklicherweise auch Husserl bediente. Von der Behandlung dieses Problems hängt nichts weniger ab als die Husserlsche Entgegnung dem Vorwurf gegenüber, die Reduktion sei (als „hyperbolisches“ Vorhaben etwa) nicht vollständig vollziehbar und daher möglicherweise als Methode sich selbst verantwortender Philosophie zu verwerfen. ${ }^{21}$ Und letztlich folgen hieraus, und allein hieraus, positiv für Husserl die „erkenntnisethischen“ Konsequenzen der Reduktion; anders gesagt: Der Anspruch seiner Philosophie muss "Motivation fïr die Verweltlichung “ sein. Was genau unter dieser Verweltlichung zu verstehen ist, soll jetzt Thema sein.

Es gilt, dieser vermeintlichen „Gegen“-Bewegung zur Reduktion eine rechte Situierung in Husserls Theorie der phänomenologischen Reduktion zu verschaffen. Wie hat nach Husserl die Reduktion in rechter Weise vonstatten zu gehen, oder was von dieser Frage untrennbar ist: wie ist in rechter Weise dieser Zuschauer zu etablieren? Freilich hört das philosophierende Subjekt (das wird Husserl nicht müde zu betonen) niemals auf, ein Mensch aus Fleisch und Blut zu sein, der in einer Lebenssituation mit ihren Ansprüchen und Erfordernissen steht. Auch scheint Husserls Konzeption der natürlichen Einstellung nahe zu legen, dass diese aus sich selbst heraus gewissermaßen nicht .gesprengt" werden kann, sondern als stets erweiterbarer, sich im Einstimmigkeitsstil aber nicht prinzipiell ändernder Horizont nie an ein Ende kommt. In der natürlichen Einstellungen kann es also selbst keine Motive geben, sie zu verlassen, jedenfalls keine aktiven."2 In dieser Hinsicht ist das Problem, wie

2" Hart, James, The Person and the Common Life. Studies in a Husserlian Social Ethics. Dordrecht/ Boston/ London 1992, Kap. 1, insbesondere S. $34 \mathrm{ff}$.

$\therefore$ Diese Kritik, die gern (und etwas leichtfertig) gegen Husserl erhoben wird. wurde im wesentlichen bereits von Husserls Assistenten Fink formuliert, der die Undurchführbarkeit der Reduktion an der Unmöglichkeit einer genuinen phänomenologischen Sprache dingfest machen möchte (Vgl. VI. CM, a.a.O., $\S 10$ u. 11, S. 93 - 169).

$\because$ Zu dieser Diskussion, worin eine Motivation für die Reduktion gesehen werden kann. vgl. Finks Anfangsstück eines systematischen Werks von 1930 und Husserls Anmerkungen dazu 
die natürliche Einstellung zu verlassen sei, gerade durch die Konzeption derselben gewissermaßen hausgemacht; andererseits geht es Husserl darum, hierdurch die Radikalität der Reduktion, die durch nichts prinzipiell (weltlich) Gekanntes antizipiert werden kann, zu illustrieren.

- Das Problem des Verlassens der natürlichen Einstellung versucht Husserl zunächst durch seine Lehre von der Ichspaltung zu lösen. Diese Spaltung spaltet das vordem einheitlich lebende Ich in ein unteres, geradehin lebendes und ein oberes, durchgängig reflexives Ich. Die vermeintliche Radikalität der Spaltung ,überspringt“ aber zunächst die Frage nach der Art und dem Vollzug des Übergangs von natürlicher in die phänomenologische Einstellung. Husserl drückt dies in einem, wie er notiert, ,reifen“ Manuskript von 1926 folgendermaßen aus: „Wesensmäßig dazugehört [sc. zur transzendentalen Reduktion], dass im Anfang der Phänomenologie die im phänomenologisierenden Subjekt stattfindende Ichspaltung den Charakter hat einer Spaltung in unteres natürlich eingestelltes und oberes in der Epoché und im subjektiven Forschen lebendes Ich." 23

Die problematische Metapher der Spaltung des Ich darf nun nicht so verstanden werden, als ob zwei voneinander unabhängige „Ichteile“ (oder gar ein ganz neues Ich) „kreiert“ würden (wie wäre das phänomenologisch auszuweisen?24), noch ist diese radikale Abspaltung mit der Reflexionsiteration zu verwechseln, die, obwohl sie einen Regress darstellt, doch nicht zu fürchten ist, weil dieser „nichts Neues“ ergibt; bildlich verstanden, ist die Iteration zu vergleichen mit einem Blick in den Spiegel mit einem zweiten Spiegel im Rücken, der dem Betrachter scheinbar endlos vervielfältigt lediglich seinen reflexiven Blick zurückwirft. So verstanden führt die Reflexionsiteration niemals über die natürliche Einstellung hinaus, wie es umgekehrt genauso absurd wäre, die natïrliche Einstellung so zu konzipieren, als ob es in ihr gar keine Reflexion (praktischer oder gar theoretischer Art) gäbe, die Sonderproblemen des alltäglichen Lebens entspräche. Die Reflexionsiteration ist also gerade deshalb nicht radikal, weil sie keine echte Spaltung des Ich bedeutet.

Die eigentliche Ichspaltung, die „mit einem Schlage“ den Zuschauer hervorbringt, ist vielmehr eine Spaltung in zwei radikal unterschiedene Einstellungen und ist somit eine Ent- oder Aufdeckung dessen, was auch treffend das

(Hua.-Dok. II/2, a.a.O., S. 10 - 105). Alle „weltlichen“ Motivationen hierzu werden von Husserl kategorisch ausgeschlossen; s. insbes. seine Anm. 114 auf S. 31.

23 B II 9/50b. Kursivierung ergänzt. Es verdient eine Erwähnung, dass Fink dieses Manuskript teilweise wörtlich in seinen Text der Einleitung zum systematischen Werk (vgl. vorige Anm.) einarbeitete (vgl. Hua-Dok., a.a.O., II/2, S. 298 - 300). Dass Fink im gleichen Text bereits „weltliche“ Motive für die Reduktion diskutiert, kann als eine Vordeutung seiner kritischen Behandlung in der VI. CM (a.a.O., von 1932) angesehen werden. kann und nicht zwei oder mehrere voneinander unabhängige. Husserl betont immer, dass im Leben im höherstufigen Strom der niedere zwar nach wie vor existiert, aber in „Inaktualität". 
„Doppelleben“ des Subjekts genannt wurde ${ }^{25}$; das Ich ist immer schon durch dieses Doppelleben konstituiert, aber es weiß nichts davon in natürlicher Einstellung. Diese Doppelung von Einstellungen meint nun nicht so sehr die Tatsache, dass das konkrete Subjekt in zwei radikal voneinander verschiedenen Einstellungsweisen leben kann - in dieser Hinsicht behauptet Husserl ja ähnliches von der künstlerischen oder religiösen Einstellung, als beide der natürlichen entgegengesetzt. ${ }^{26}$ Sondern in dieser, die Einheitlichkeit des natürlichen Ich erst aufspaltenden Entfernung von und gleichzeitig reflexiven Rückwendung auf sich selbst entdeckt das Subjekt die transzendentale Subjektivität als Sphäre von Leistungen, in welchen sich die Welt für das erfahrende Bewusstsein konstituiert.

Dieser Vorgang ist nun nicht allein ein Enthüllen der Region der transzendentalen Subjektivität als neuen thematischen Bereichs phänomenologischer Forschung. Er motiviert auch die methodologische Einsicht, dass ich selbst es bin, der als transzendentales Subjekt diese Welt für mich selbst konstituiert, zunächst in Anonymität des um sich selbst und seine Leistungen nicht wissenden Lebens natiirlicher Einstellung. Die Reduktion hebt aber diese Anonymität gerade auf. Das Ich ist immer schon ein Doppeltes, ohne in der natürlichen Einstellung darum zu wissen: ein anonym leistendes und konkret lebendes, konstituiertes aber gleichzeitig konstituierendes. Die Entdeckung des Transzendentalen ist also nicht nur die Eröffnung einer neuen, dem Typus nach ungekannten Forschungsregion (das hieße, sie unterzubestimmen; denn sie kann nicht bloß eine Region neben anderen (weltlichen) Regionen sein), sondem die Reduktion enthüllt gerade die natürliche Einstellung als Stätte immer schon vor sich gehender Leistung der Weltkonstitution. Das konfundiert nicht die natürliche Einstellung mit der transzendentalen, aber macht die natürliche Einstellung erst transzendental verständlich, insofern ihr transzendentaler Charakter ans Licht kommt.

Gegenüber der „,negativen“ Darstellung der Epoché (im Cartesianischen Weg), derzufolge nach Einklammerung der Welt ein ,reines Ich“ als „Residuum der Weltvernichtung “ übrigbliebe, das zudem noch als ein , letztes Endchen Welt" aufgefasst werden könnte, betont Husserl zunehmend den positiien Sinn der Reduktion, die recht verstanden ein radikales ,.Durchbrechen ion

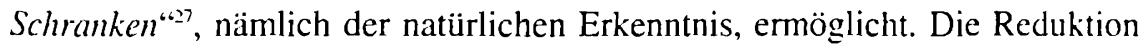

\footnotetext{
Vgl. Bernet, Rudolf, „Phenomenological Reduction and the Double Life of the Subject”. a.a.O.

2h Vgl. den Brief an von Hofmannsthal (Hua-Dok., a.a.O., IIV7. S. 133 - 36). worin Husserl die phänomenologische und die ästhetische Einstellung kontrastiert. sowic Husserls Rezension der Übersetzung der Reden Buddhas, worin er die ..Religiosität" des buddhistischen Denkens eine ..in Schau und ringender Tat rein nach innen gewandte - ich möchte sagen. eine nicht .transzendente", sondern ,transzendentale " - (Aufsätze und Vorträge. Hua XXVVII. S. 125f.) nennt. Allerdings sind Kunst und Religion darin radikal von der Phänomenologie verschieden, dass sie keine strenge Wissenschaftlichkeit erstreben.

3 Kern. Iso, ,.Die drei Wege zur transzendental-phänomenologischen Reduktion in der Philosophie Edmund Husserls", in: Tijdschrift voor Filosofie, Bd. 24 (1962), S. $303-49$.
} 
bewirkt also gerade keine Verarmung oder Vereinzelung des Erkenntnissubjekts, das sich mit seinem eigenen (primordialen) Ego alleingelassen sieht, sondern eine Bereicherung des Erkenntnisfeldes um ,unendliche“ Horizonte gegenüber dem endlichen Horizont der in der natürlichen Einstellung durchlebten Um-, bzw. „Heimwelt“.

Ist diese nun enthüllte transzendentale Sphäre weltkonstituierend, so kann umgekehrt gesagt werden, dass die Welt erst dann vollständig als Welt (phänomenologisch als sinnhafter Horizont von Verweisungsbezügen) erkannt wird, wenn sie als Leistungsgebilde transzendentaler Vollziige erkannt wird. Husserls Transzendentalphilosophie ist im emphatischen Sinne Erkenntnis von Welt, wenn von Sein nur als Konstituiertsein-für ein menschliches Bewusstsein und nur von diesem Für-uns-Sein als wahrem Sein gesprochen werden kann, weil es ein anderes Sein( für uns) nicht geben kann. Die aus transzendentaler Forschung gewonnenen Erkenntnisse führen somit nicht hinter die Welt zurück in eine "Hinterwelt" (oder, was schlimmer wäre, über die Welt hinaus), sondern in rechtverstandener Weise geradewegs in die Welt hinein, bzw. zu einem rechten Verständnis von ihr; denn sie konnte vordem als solche gar nicht thematisch werden, wenn natüliche Einstellung besagt, dass die Welt als Welt (und d.i. als Universalhorizont) nie thematisch wurde, sondern nur Dinge oder Sachverhalte in ihr, sofern das naturliche Ich stets irgendwelchen Sonderinteressen und -zielen hingegeben, also „weltbefangen“" war. Insofern sind die „Erkenntnisse“ der transzendentalen Wissenschaft nicht solche, die von einer ,verkehrten Welt“ zeugten, im Gegenteil; das Verhältnis der transzendentalen zu den weltlichen, positiven Wissenschaften und letztlich das Verhältnis von Phänomenologie und Welt charakterisiert Husserl (in einem Manuskript aus dem Jahre 1931) folgendermaßen: „Die transzendentale Wissenschaft [...] stellt sich nicht neben die natürlich-naive, die positive Weltwissenschaft, sie umgreift radikal und universal in der Weise der transzendentalen (4) Aufklärung die Welt mit, [die Welt] somit <mit> allen Wissenschaften [...]. Sie enveitert nicht den natürlichen Sinn der Welt, wenn sie der Welt transzendentalen Sinn gibt, oder vielmehr [wenn sie] ihren transzendentalen Sinn, den sie im Verborgenen hat, ans Licht bringt." ${ }^{\text {"28 }}$

Die Konstitutionstheorie ist also keinesfalls mit einer Zweiweltentheorie zu verwechseln, die einen neuen Dualismus zwischen transzendentaler und natürlicher Welt proklamierte: Die transzendentale Phänomenologie ist gerade der Welt zugewandt, unablösbar auf sie bezogen in einer Weise, dass sie sie als solche thematisiert, damit aber erst entdeckt. ${ }^{29}$ Damit wird die Welt aber

28 B II $7 / 71 \mathrm{~b}$, Kursivierung ergänzt.

29 Vgl. auch folgendes Zitat: „Dieses Ichsein und Ichleben, in dem und durch dessen besondere Leistung und Leistungsstruktur, Welt " als für mich es seiend und mit ihrem ganzen Sinngehalt sich konstituiert, ist nicht selbst weltlich Reales, es ist an sich früher als Welt überhaupt und alles einzelnen weltichen Seienden. Andererseits ist diese Welt als aus seiner Leistung Seinssinn empfangend nichts von ihm, dem absoluten Ich und Ichleben Getrenntes, nicht etwas neben ihm und zu ihm Beziehung Habendes. Es hat zu ihm Beziehung als in ihm Konsti- 
gleichzeitig in ihrem Sein enveitert. Der, metaphorisch gesprochen, ,zweidimensionale" Welthorizont wird durch eine dritte, eine Tiefendimension erweitert, die erst die Welt als das verständlich macht, was sie für den in ihr (als Objekt unter anderen Objekten) lebenden Menschen ist, der aber zugleich ein Subjekt fiir die Welt ist. Welt wird somit in phänomenologischer Betrachtung ein aus ,,intersubjektiver Synthesis konstituierte[s] Gebilde-Universum, derart, dass darin auch jedes transzendentale Ich durch ein Selbstgebilde, durch die intersubjektiv apperzipierte und apperzipierbare (erfahrbare) Bildung IchMensch vertreten ist".

Wie bereits gesagt, ist „Verweltlichung“ der Begriff, den Husserl für diesen Vorgang des sich in die Welt Zurücklegens des Transzendentalen verwendet. Um diese Gegenbewegung zu verstehen, muss gefragt werden, was nun mit dem transzendentalen Zuschauer geschieht, der diese Prozesse enthüllt und für sich und Andere in seinem Gestalten der transzendentalen Wissenschaft zugänglich macht. Hierzu muss der Sinn dieser Welterweiterung oder entgrenzung genauer geklärt werden; denn wo spielt sich diese Erweiterung in phänomenologischer Rückfrage eigentlich ab? Auch wenn die Reduktion erst einen unverstellten Blick auf die Welt freigibt, so kann dieser Ruick-Schritt als methodisches Verfahren doch nur ein solcher vom phänomenologisierenden Ego her und auf dieses zurück sein. Reduktion ist ,wesenhaft“ zunächst eine Reduktion auf mein Ego (als phänomenologisierendes), oder sie ist überhaupt nichts: „Phänomenologe sein, das ist etwas, das nur der Phänomenologe erfahren und erkennen kann. ${ }^{\text {“31 }}$ Auch wenn die "Sphäre“ der transzendentalen Subjektivität die des Ich in eidetischer Allgemeinheit ist, so ist doch die sich hier bildende Erkenntnis (und a fortiori die Bildung einer transzendentalen Wissenschaft) je eine Tätigkeit eines konkreten Ego und gehört ,zu jeder menschlichen Seele als Möglichkeit"“32, auch wenn die Institution „Wissenschaft“ letztlich ein in gemeinsamer Bemühung konstituiertes intersubjektives Gebilde mit einer historischen Entwicklung ist. Die transzendentale Enthüllung der Welt spielt sich also gewissermaßen als Erkenntnis ,im Kopfe des Phänomenologen" ab und ist, trotz ihrer „Höherstufigkeit“ gegenüber Verhaltensweisen in natürlicher Einstellung, eine menschliche Tätigkeit. Ist im Sinne der Konstitutionstheorie alles Verhalten (Wahrnehmen, Erfahren) ein Konstituieren, so muss also auch diejenige Tätigkeit, die die Konstitution der Welt enthüllt. selbst eine solche Konstitution sein, insofern sie den Erfahrungsbestand in der Welt selbst erweitert. Dieser einfache, aber entscheidende Gedanke eröffnet den Weg zur Verweltlichung.

Jedoch muss auch bei der eigentümlichen Aktivität des Phänomenologen unterschieden werden zwischen intentionalem Akt und dessen Inhalt: Ist der

tuiertes zum Konstituierenden, und diese Beziehung liegt ganz und gar innerhalb der absoluten. der transzendentalen Subjektivität.“ (B II 4/82a/b, von 1929/30)

B II 4/48b, undatiert (wohl um 1932).

B I 5/156a, von 1930.

So Husserl in: VI. CM (a.a.O., S. 189). 
Inhalt der phänomenologisierenden Akte ein idealer, transzendentaler, so ist der Vollzug dieser Akte immer ein faktischer eines Menschen, sofern er sich psychisch (in diesen Fall wissenschaftlich) betätigt. So ist diese Konstitution zwar eine „höherstufige“, aber doch "gegenüber der ,naiven“ der um sich selbst unwissenden der natürlichen Einstellung ${ }^{33}$ immer noch eine Konstitution. Das in ihr aktmäßig Erzeugte išt demgemäss nichts Transzendentales, sondern Mundanes oder, wie sich Husserl ausdrückt, „Psychologisches“"34: „Indem Ich, der Phänomenologisierende, die Konstitution der Welt (als Seinssinn des transzendentalen Ego) erforsche, vollziehe ich damit eine Fortkonstitution der Welt selbst, nämlich durch Psychologisierung der transzendentalen Erlebnisse, die als weltkonstituierende fungieren. [...] Alles [transzendental Erforschte] psychologisiert sich, legt sich dadurch der Welt ein, als den menschlichen Seelen zuzurechnen. ${ }^{\text {“35 }}(1933 / 34)$

Dieses gewissermaßen ,automatisch“ durch erfahrend-erkennende Enthüllung sich vollziehende „Zurücklegen“" in die Welt als Psychologisierung ist der prägnante Sinn von „Verweltlichung“. Hier muss :aber allgemein gelten: Alles Konstituieren ist Verweltlichung im beschriebenen Sinne, wie korrelativ Welt selbst dadurch einen umfassenderen Horizont erhält, indem sie immer mehr ,angereichert“" wird durch die Tätigkeit des Phänomenologen, der in seiner Forschungstätigkeit „die Welt selbst fortkonstituiert“, d.h. um neue Sinnbestände erweitert. Das Transzendentale ,,lokalisiert sich“" in der Welt, genauer in ,diesem Menschen“, der phänomenologisiert, ohne aber aufzuhören, transzendental zu sein. Im Sinne des gebrauchten Gleichnisses: die neue, dritte Dimension ,verschmilzt“ nicht mit der Zweidimensionalität der Weltoberfläche, sondern behält ihren Status als dritte Dimension. . .

Ist dies so, drängt sich sogleich die Frage auf, wie es dann mit der Unbeteiligtheit des Zuschauers steht. Auch wenn der Phänomenologe die partikularen Interessen der jeweiligen Situationen praktischer Existenz inhibiert, also uninteressiert ist im Sinne eines unmittelbaren, ,handanlegenden“ Beteiligtseins (wenn er auch als Forscher an der Welt selbst eminentes Interesse und Neugier hat), so kann doch der an den Phänomenen arbeitende und somit konkrete Forschungsleistung hervorbringende transzendentale Wissenschaftler nicht gut als unbeteiligt bezeichnet werden. In diesem Sinne korrigiert sich Husserl folgerichtig, wenn er selbstkritisch erwägt: „Ich bin der transzendentale ,Zuschauer' (das ist kein zureichender Ausdruck); ich bin der Phänomenologisierende,

$33 \mathrm{Vgl}$. B II $7 / 71 \mathrm{~b}$. Hier setzt Husserl geradezu ,Welt als. Welt der natürlichen Einstellung gleich mit "naiver Konstitution“.

34 Husserl wendet sich in diesem Zusammenhang auch gegen das falsche Verständnis der transzendentalen Phänomenologie als Psychologie bzw. als "transzendentaler Psychologismus“ (Vgl. Husserl, Edmund, Formale und transzendentale Logik, (Hua XVII), hg. von P. Janssen. Den Haag 1974, § 99).

3 VI. C.M, (a.a.O., S. 213. Kursivienung ergänzt).

and 
der alles, was ich selbst bin, enthïllt und damit zum wahrhaften, zum erkenntnismäßig Seienden macht. “.36 (1933/34)

Diese späte Einsicht Husserls stellt sein ursprüngliches Konzept des unbeteiligten Zuschauers in Frage, es steht aber dennoch in völligem Einklang mit seiner Vision von den Philosophen als den „Funktionären der Menschheit“, die die tiefsten Wahrheiten der Menschheit für die Menschheit enthüllen und die damit den radikal-aufklärerischen Auftrag, an dem Husserl mit seiner Philosophie bis zuletzt festhielt, erfüllen, und zwar eine Philosophie, die als der eigentliche Zwecksinn der Menschheit darauf abzielt, ein ,höheres Menschheitsleben" zu ermöglichen oder zumindest konkret auf den Weg zu bringen. Dies ist jedoch nur möglich, wenn plausibel gemacht werden kann, dass die transzendentale Einstellung des Phänomenologen zwar nicht bruchlos aus der natürlichen Einstellung hinats-, wohl aber nach Vollzug der Reduktion wieder in sie ,zurückführt“, was aber diesem Vorgang insofern unangemessen ist, als nach der Reduktion erkannt ist, dass die transzendentale Einstellung nichts anderes als eine Fortkonstitution der natürlichen ist. Anders gesagt: Natürliche Einstellung ist immer schon um sich unwissende transzendentale Einstellung; transzendentale Einstellung ist nichts anderes als „erwachte“ natürliche Einstellung. Oder noch anders: transzendentale Einstellung ist das Erste tê phlísei, die natürliche das Erste pròs hemãs. Da Phänomenologe zu werden für jedes vernünftige Wesen eine Möglichkeit darstellt, ist die Forderung, die Menschheit zur Phänomenologie anzuleiten, also radikal aufzuklären, nur konsequent. Oder um es mit den Worten Finks zu formulieren: Die Phänomenologie hat einen „transzendental-pädagogischen “ Auftrag. ${ }^{37}$

Das Gegenbild zu diesem ,aufklärerischen“ Ideal wird von Husserl auch erwogen; man kann dies eine uneigentliche Verweltlichung oder Rückkehr in die naiv'natürliche Einstellung nennen, und sie zeigt, wie Husserl das Verhalten des Philosophen gerade nicht verstanden wissen möchte. Husserl kommt in den Zusammenhängen darauf zu sprechen, wenn er vom Phänomenologisieren als einer beruflichen Einstellung unter anderen spricht, die man zeitweise betätigen, aber dann wieder verlassen und sich wieder dem ursprünglichen Lebensstrom der ursprünglichen Naivität hingeben kann. ${ }^{38}$ Von dieser Perspektive aus gesehen, verhält sich der transzendentale Wissenschaftler nicht anders

is VI. CM (ebd., S. 192. Kursivierung ergänzt).

3 Vgl. VI. CM, (ebd., S. 110). Auch wenn (oder gerade weil) Husserl eine ..transzendentalen Pädagogik" nicht selbst ausführt, so lässt er diesen Begriff in der ansonsten heftig annotierten VI. CM seines Assistenten kommentarlos ,durchgehen“.

¿s Vgl. B I 5/155a: „Hier wird es darauf ankommen, den Wechsel der Einstellungen zu verstehen. der mit der phänomenologischen Reduktion in meiner Freiheit steht, zu verstehen das Sichzurïckversetzen in die natürliche Einstellung und zwar als ,Rückkehr', wie wenn ich die phänomenologische Arbeit ruhen lasse und mich den Steuersorgen dieser Zeit hingebe. oder mit den Kindern mich unterhalte etc. Die transzendentale Epoché ist eine theoretisch erfahrende und so überhaupt eine wissenschaftliche Einstellung, und wie eine wissenschaftliche Einstellung überhaupt kann ich sie verlassen und in außer-wissenschaftliche eintreten." (von Ende 1931). 
als jeder andere Wissenschaftler auch, ,in der Alltäglichkeit““. ${ }^{39}$ In einer solchen Beschreibung eines Wissenschaftlers unter den „,normalen Menschen“ unterscheidet sich der Phänomenologe aus der Sicht der natürlichen Einstellung in nichts von jedem anderen positiven Wissenschaftler. Jedoch nur diese Perspektive zu betrachten, hieße, sich wiederum blind gegenüber der transzendentalen Dimension zu stellen und damit den Auftrag der Phänomenologie zu verkennen, und das hieße, ihr verantwortungslos zuwiderzuhandeln. Man missachtete gerade deshalb diesen Auftrag, weil man Phänomenologie zu betreiben als Beruf unter anderen Berufen ansähe. Wie aber wird ein Beruf unter anderen normalerweise ausgeübt?

Wie man im natürlichen Leben ständig bestimmte Partial-,,Epochaí “ übt (das einfachste Beispiel ist etwa zuwendende Aufmerksamkeit einer Sache gegenüber unter Ausblendung einer anderen, und in dieser Hinsicht wäre ein Beruf ein temporäres Ausblenden anderer Lebenszusammenhänge während der „Berufszeit"), so ist eine solche ,abstraktive Abblendung“ jederzeit möglich, wenn auch nicht „korrekt“" im Sinne des vom Phänomenologen Geforderten; denn der Phänomenologe würde seinen Auftrag verkennen, wenn er sich mit der Auffassung begnügen könnte, nach seiner Arbeitszeit wiederum ,,in die Alltagswelt zurückzukehren" und so zu tun, ,als wäre nichts gewesen“. . $^{0}$ Eine solche Haltung wäre für den, der Selbstverantwortung und Verantwortung für die Menschheit gerade auf sein Schild erhoben hat, in eklatantem Maße unverantwortlich. Man kann eine solche Abstraktion auch als mundane oder vernveltichende (Partial-)Epoché bezeichnen. Wer auf diese Weise in die natürliche Einstellung zurückkehrt, als wäre nichts gewesen, nimmt seine Verantwortung als philosophischer Wissenschaftler nicht ernst; denn der Philosoph hat keinen Beruf neben anderen, sondern er fühlt sich zum „Funktionär“ berufen. " Daher ist die Charakterisierung des. Phänomenologisierens als Berufs unter anderen Berufen mit besonderer Vorsicht żu genießen.

Dieses Gegenideal gegenüber der rechtverstandenen Reduktion, die ihre Verweltlichung bereits in sich trägt, macht deutlich; dass eine eigentliche „Rückkehr“ mitnichten ein Zurückgehen in einen dunklen (Ur-)Zustand ist, sondern dass die Reduktion mit einem Schlage alles verändert, der ein leichtfertiges Rückkehren für immer unmöglich macht. Deshalb ist auch Husserls bekannter, ohne diesen Hintergrund unverständlicher Vergleich mit der ,religiösen Umkehrung" der Sache angemessener, insofern die Welt zwar die gleiche bleibt, für den philosophisch Aufgeklärten (im gleichen Sinn wie für den

39 Vgl. VI. CM, (a.a.O., S. 143, Anm. 469). Diese Form der Interaktion mit Anderen fungiert bei ihm bezeichnenderweise als „2a“ der möglichen Interaktionsformen; „, " “ ist der „Verkehr [der Phänomenologen] miteinander (ebd.), ,2“ „Die Phänomenologen im Verkehr mit den Nicht-Phänomenologen, sie zur phänomenologischen Reduktion und zum Nachverstehen der Phänomenologie anleitend" (ebd).

$\therefore$ Vgl. VI. CM, (ebd., S. 191; Anfang von Beilage VI, von 1933 oder 1934).

1 Vgl. Husserl, Krisis, (Hua VI), a.a.O., S. 15. Husserl spielt hier selbst auf die Grundbedeutung des Wortes .,Beruf“ an. 
religiös Bekehrten) aber einen neuen Sinn angenommen hat, der nicht mehr aufgegeben werden kann (Hua VI, S. 140). Gleichzeitig ist klar, dass Husserls Entwurf nicht in eine (quasi- oder pseudo-)religiöse Haltung führen darf, derzufolge man sich der Welt zugunsten einer höheren „Welt“ abwenden müsste. ${ }^{+2}$ Die Reduktion führt für den Phänomenologen gerade zu einem rechten Zugang zur Welt, einer Welt, die aber nun mit dem Index des Unendlichen ausgestattet ist. In diesem Verständnis von Welt ist das durch den Phänomenologen enthüllte Transzendentale nichts anderes als, uneigentlich gesprochen, ein „Bestandteil“" der Welt selbst, kein Vorsein und kein Me-On. Bezeichnet Husserl nun dieses so universal verstandene Transzendentale als das Absolute, so ist die Welt als Leistungsprodukt des Absoluten selbst (wieder uneigentlich) ein „Teil“, oder wie Husserl sich ausdrückt, ein „Korrelat“ des Absoluten: „Im transzendentalen Totalfeld des im absoluten Sinn Seienden tritt die natürlich-naive Welt, die der <der> Transzendentalität noch nicht bewusst gewordenen Menschen, als Korrelat auf, sie ist mit diesem ihr zugewachsenen Seinssinn selbst Moment im Absoluten. “43 (von Januar 1934)

Als „Moment im Absoluten“ als strömender Tendenz der Weltverwirklichung ist die Welt (als geschichtliche Welt) nichts anderes als absolutes Faktum als dasjenige, worin alle Leistungen terminieren und sich geschichtlich sedimentieren. Gemäß dem universalen Korrelationsapriori ist weder die Transzendentalität allein relativ auf die Welt noch umgekehrt, sondern sie sind beide relativ aufeinander. ${ }^{+4}$ Das Absolute wird aber immer nur bewusst „kommt“ (in der Sprache spekulativer Philosophie) „,zu sich selbst“ - in einem konkreten Ich, das, leibhaft räumlich-zeitlich lokalisiert in der Welt, selbst sein absolutes Hier nie aufgeben kann und somit für sich selbst ein absolutes Faktum als „Index“ des Absoluten ist. Den Urquellpunkt dieses im Menschen als Mensch lokalisierten Weltbewusstseins sieht Husserl in seinen späten Reflexionen auf die Seinsweise des transzendentalen Ego in der stehend-strömenden „lebendigen Gegenwart", die weder eigentlich als ichhaft zu bezeichnen ist, noch voll und ganz der Welt „gehört“, sondern der absolute Erfahrungspulsschlag ist, an dem sich Welt für den Menschen offenbart, für je mich, ,in mei-

42 Das ist genau Husserls Kritik am ,,buddhistischen“ Ideal der Weltflucht, deren rückwärtsgewandte Innenbewegung ansonsten durchaus mit der ,.sokratischen“ Bewegung verglichen werden kann, die aber jener gegenüber dazu angestellt wird, un gerade die rechten Prinzipien und Motive der Handlung in der Welt zu erwägen. In diesem Sinne ist der Buddhismus ..verantwortungslos“. Vgl. hierzu den Text ,Sokrates und Buddha“ rom Frühjahr 1926, in B I $21 / 88$ - 94. Vgl. hierzu auch von Karl Schuhmann ,Husserl and Indian Thought”, in: Phenomenology and Indian Philosophy (hg. von D. P. Chattopadhaya, L. Embree, J. N. Mohanty, Neu Delhi 1993, S. 20 - 43). Schuhmann zitiert aus diesem unveröffentlichten Manuskript und legt eine ausführliche Deutung desselben vor.

43 So Husserl in: VI. CM, (a.a.O., S. 215 ; vom 21.1.1934).

4 Im Sinne von Husserls Ausführungen in $\S 51$ der Krisis könnte man auch sagen, dass sie beide ineinander und wechselseitig fundiert sind (Hua VI, S. 177). 
nem Faktum, mit meiner lebendigen Gegenwart, die ich faktisch lebe, apodiktisch vorfinde und mit allem, was darin enthüllbar liegt." (von 1935). ${ }^{45}$

„Apodiktisch“ sei das Stichwort für eine abschließende Überlegung. Das absolute Faktum meines letztfungierenden Ich, das ich im Phänomenologisieren enthülle, muss nach dem Zitierten auch ein apodiktisches sein. Erkenntnis des Absoluten (was nicht zu verwechseln ist mit absoluter Erkenntnis) ist daher immer zurückbezogen auf ein Subjekt, das in sich das Absolute als „Alleinheit" von Transzendentalem und Welt enthüllt, das also nichts anderes tut, als dieses sein Doppelleben thematisch zu machen als in seiner apodiktischen erfahrenen Faktizität lokalisiert. ${ }^{46}$ So kann man abschließend die These wagen, dass das Absolute im phänomenologischen Sinn unmittelbar mit der Frage steht und fällt, wie es sich zum apodiktisch reduzierten Ursein (wie Husserl die lebendige Gegenwart auch nennt) verhält. Die Reichweite absoluter Erkenntnis lässt sich letztlich möglicherweise mit dem Umfang an apodiktischer identifizieren. Die Frage wäre dann, ob nicht beide, das Absolute und Apodiktizität, zusammengehören müssen, will man letztlich nicht in eine spekulative oder gar mystische Betrachtungsweise verfallen, sondern wenn man auf phänomenologisch sinnvolle und das heißt ausweisbare Weise das Absolute soll thematisieren können und damit Husserls Version von Transzendentalphilosophie treu bleiben kann. In diesem Sinn stellt er sich in einem späten Manuskript die Frage, die zu beantworten seinen Interpreten aufgegeben ist: „Wie expliziert sich systematisch die Apodiktizität des Ich, was ist ihm, als sich Besinnenden, als sich im wachen Leben als Weltleben Findenden, zueigen, was ist, wenn Ichapodiktizität und Weltapodiktizität äquivalent sind, als Korrelate in der Weltapodiktizität das wirklich Apodiktische? “47 (von 1935)

45 K III 12/25b (1935). Vgl. ebd.: „Aber ich [...] bin apodiktisch das Ich der faktischen Wirklichkeit und ich bin das Ich der Vermögen, die ich insbesondere als eidetisch denkendes und sehendes Ich mir faktisch erworben habe."

Vgl. C 17/64a: „Aber hier ist zu bedenken, dass die rückfragende transzendentale Enthüllung des ursprünglichen Lebens eine Leistung des transzendental phänomenologisierenden Ich ist. und Rückfrage ist von der konstituierten Welt aus, die das Ich als weltlich konstituiert enthält.“ (um 1932).

B II $21 / 18 b$. 